\title{
Ruta de apropiación de las TIC para profesores de educación superior fundamentada en el modelo Tecnológico Pedagógico Disciplinar
}

Diana Patricia Ospina-Pineda ${ }^{1}$

Universidad de Antioquia

dianaospina4@gmail.com

Ángela María Valderrama-Muñoz ${ }^{2}$

Universidad de Antioquia

angela.valderrama@udea.edu.co

María Mercedes Jiménez-Narváez

Universidad de Antioquia

maria.jimenez@udea.edu.co

Vanessa Arias-Gil ${ }^{4}$

Universidad de Antioquia

vanessa.arias@udea.edu.co

Marisol Lopera-Pérez ${ }^{5}$

Universidad de Antioquia

marisol.loperap@udea.edu.co

Herlaynne Segura-Jiménez ${ }^{6}$

Universidad de Antioquia

herlaynne.segura@udea.edu.co

DOI: https://doi.org/10.21158/2357514x.v8.n2.2020.2721

Cómo citar este artículo: Ospina-Pineda, D. P.; Valderrama-Muñoz, Á. M.; Jiménez-Narváez, M. M.;

Arias-Gil, V.; Lopera-Pérez, M. Segura-Jiménez, H. (2020). Ruta de apropiación de las TIC para profesores

de educación superior fundamentada en el modelo Tecnológico Pedagógico Disciplinar. Revista Virtu@lmente, 8(2), 87-99.

DOI: https://doi.org/10.21158/2357514x.v8.n2.2020.2721

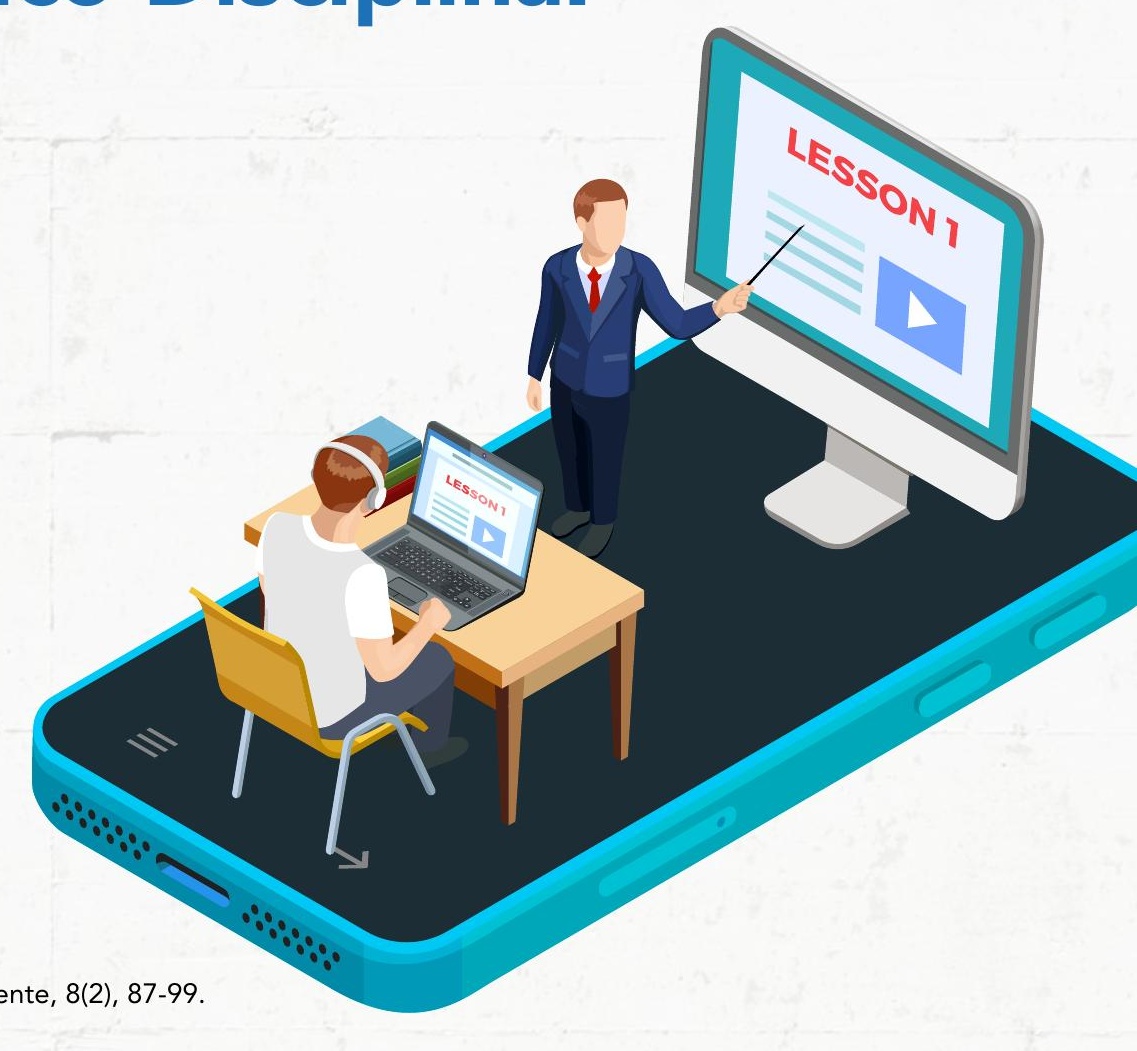

Fecha de recepción: 08 de agosto 2020

Fecha de aprobación: 11 de septiembre 2020

${ }_{1}^{1}$ Magíster en Literatura Colombiana - Universidad de Antioquia. ORCID: https://orcid.org/0000-0002-4806-5116

${ }^{2}$ Magister en Administración de Negocios - Universidad EAFIT. ORCID: https://orcid.org/0000-0001-5714-2884

${ }^{3}$ Doctora en Educación - Universidad de Antioquia. ORCID: https://orcid.org/0000-0002-7402-4393

${ }^{4}$ Magíster en Educación en Ciencias Naturales - Universidad de Antioquia. ORCID: https://orcid.org/0000-0002-9449-6144

${ }^{5}$ Doctora en Investigación transdisciplinar en Educación - Universidad de Valladolid: Valladolid, Castilla y León, España. ORCID: https://orcid.org/0000-0002-2548-6516

${ }^{6}$ Máster en Comunicación Digital - Universidad de Vic. Magíster en Sociedad de la Información y el Conocimiento - Universidad Oberta de Cataluña. ORCID: https://orcid.org/0000-0002-2988-9179 


\section{RESUMEN}

Este artículo sintetiza el diseño de una ruta de apropiación de las Tecnologías de la Información y la Comunicación (TIC), fundamentada en el modelo Tecnológico Pedagógico Disciplinar (TPACK por su sigla en inglés, Technological Pedagogical Content Knowledge), que busca generar en los profesores de educación superior reflexiones y acciones para cualificar sus prácticas docentes en la modalidad virtual. La ruta se realizó en el marco de un proyecto que utiliza la metodología de investigación basada en diseño. Así las cosas, el objetivo de este artículo es reconocer los elementos estructurantes tenidos en cuenta durante el diseño, retomando para ello algunos aspectos identificados en la literatura y los relativos a la identidad del ser docente universitario, así como el modelo TPACK, las propuestas basadas en las e-actividades, el trabajo colaborativo y la gamificación. Finalmente, se concluye que el diseño educativo de una ruta de apropiación de TIC implica una reflexión a fondo sobre la relación entre lo disciplinar, lo educativo y lo tecnológico que se pregunte sobre cuáles son las transformaciones que acontecen en la educación cuando se utilizan las TIC; pues queda claro que el diseño de e-actividades ha significado un aporte para repensar la educación en ambientes virtuales de aprendizaje, ya que permiten pasar de una acción expositiva hacia formas más dialógicas e interactivas

Palabras clave: apropiación de tecnología; modelo Tecnológico Pedagógico Disciplinar; Technological Pedagogical Content Knowledge; educación virtual; prácticas docentes; e-actividades; gamificación; ambientes virtuales de aprendizaje. 


\section{Route of appropriation of the ICT for teachers of higher education based on the Technological Pedagogical Discipline model}

\section{ABSTRACT}

This article synthesizes the design of a route of appropriation of Information and Communication Technologies (ICT), based on the Technological Pedagogical Content Knowledge (TPACK) model, which seeks to generate reflections and actions in higher education teachers to qualify their teaching practices in the virtual modality. The route was carried out in the framework of a project that uses the research methodology based on design. Thus, the aim of this article is to recognize the structuring elements that were taken into account during the design, taking up some aspects that have been identified in the literature and those related to the identity of university teachers, as well as the TPACK model, the proposals based on e-activities, collaborative work, and gamification. Finally, the paper concludes that the educational design of an ICT appropriation route implies an in-depth reflection on the relationship among the disciplinary, educational, and technological spheres that ask about the transformations that take place in education when the ICTs are used; since it is clear that the design of e-activities has meant a contribution to rethink education in virtual learning environments, as they allow to move from an expository action towards more dialogical and interactive ways.

Keywords: appropriation of technology; Technological Pedagogical Disciplinary Model; Technological Pedagogical Content Knowledge; virtual education; teaching practices; e-activities; gamification; virtual learning environments. 


\section{Percurso de apropriação das TIC para professores do Ensino Superior com base no modelo Tecnológico Pedagógico Disciplinar}

\section{RESUMO}

Este artigo sintetiza o desenho de um percurso de apropriação das Tecnologias de Informação e Comunicação (TIC), com base no modelo Tecnológico Pedagógico Disciplinar (TPACK pela sigla em inglês, Technological Pedagogical Content Knowledge), que busca gerar nos professores de Ensino Superior reflexões e ações para qualificar suas práticas docentes na modalidade virtual. O percurso foi realizado no âmbito de um projeto que utiliza a metodologia de pesquisa baseada em desenho. Assim, o objetivo deste artigo é reconhecer os elementos estruturantes levados em consideração ao longo do desenho, levando em consideração alguns aspectos identificados na literatura e aqueles relacionados à identidade de ser professor universitário, bem como o modelo TPACK, as propostas baseadas nas eatividades, trabalho colaborativo e gamificação. Finalmente, conclui-se que o desenho educacional de um percurso de apropriação das TIC implica uma reflexão aprofundada sobre a relação entre o disciplinar, o educacional e o tecnológico, que indaga quais são as transformações que ocorrem na educação com o uso das TIC, pois fica evidente que o desenho de e-atividades tem contribuído para repensar a educação em ambientes virtuais de aprendizagem, pois permitem passar de uma ação expositiva para formas mais dialógicas e interativas.

Palavras-chave: apropriação de tecnologia; modelo Tecnológico Pedagógico Disciplinar; Technological Pedagogical Content Knowledge; educação virtual; práticas docentes; e-atividades; gamificação; ambientes virtuais de aprendizagem. 


\section{Modes d'appropriation des TIC pour les professeurs de l'enseignement supérieur au travers d'un modèle technologique, pédagogique et disciplinaire}

\section{RÉSUMÉ}

Cet article synthétise la conception d'un parcours d'appropriation des Technologies de I'Information et de la Communication (TIC) basé sur le Modèle Technologique Pédagogique Disciplinaire (TPACK - Technological Pedagogical Content Knowledge) qui prétend générer actions et réflexions de la part des enseignants de l'enseignement supérieur dans la qualification de leurs pratiques pédagogiques en modalité virtuelle. Le parcours d'appropriation a été réalisé dans le cadre d'un projet utilisant une méthodologie de recherche basée sur la conception. Cet article a pour objectif de faire connaître les éléments structurants pris en compte lors de la conception du parcours d'appropriation en reprenant certains aspects identifiés dans la littérature et liés aux fondements de l'identité des professeurs universitaires, au modèle TPACK, aux propositions relatives aux activités virtuelles, au travail collaboratif et à la gamification. En conclusion, la conception pédagogique d'un parcours d'appropriation des TIC implique une réflexion approfondie sur la relation entre discipline, éducation et technologie, et pose la problématique de la transformation de l'éducation orientée par les TIC. La conception d'activités d'apprentissage numérique a contribué à repenser l'éducation en modalité virtuelle en passant d'une action explicative à des formes plus dialogiques et interactives.

Mots-clés: appropriation de la technologie; Modèle Disciplinaire Pédagogique et Technologique; connaissance des contenus pédagogico-technologiques; éducation virtuelle; pratiques d'enseignement; activités virtuelles; gamification; environnements d'apprentissage virtuels. 


\section{Introducción}

En el marco de una investigación regional financiada por Colciencias y la empresa privada (Jiménez-Narváez et al., 2019), se diseñó una ruta de apropiación de TIC dirigida a docentes de educación superior del Departamento de Antioquia - Colombia-, quienes, en su mayoría, son expertos en un área de saber disciplinar, pero no poseen formación docente, para contribuir a la cualificación de sus prácticas pedagógicas en la modalidad virtual. Con esta ruta se busca trascender la idea instrumental del uso de las Tecnologías de la Información y la Comunicación (TIC), así como repensar las prácticas para que los conocimientos pedagógicos, didácticos, comunicacionales y tecnológicos ocupen un lugar preponderante, tal como lo propone el modelo TPACK Tecnológico Pedagógico Disciplinar (Koehler, Mishra y Cain, 2015), así como las tendencias para el aprendizaje digital, en cuyo centro no están los contenidos sino las actividades tanto individuales como colaborativas, con el fin de potenciar la participación, la motivación y el diálogo de saberes y de experiencias que enriquecen la reflexión sobre la práctica docente.

El objetivo de este artículo es reconocer el proceso de diseño de la ruta de apropiación de TIC para los docentes universitarios de las áreas de la salud y la ingeniería en la modalidad virtual, donde se aborde la pregunta por lo pedagógico y didáctico, lo tecnológico y lo disciplinar. Para esto se presentan el análisis y la manera de abordar los conocimientos derivados del modelo TPACK, la forma de integrar los estándares de competencia, la taxonomía de Bloom y los avances en el desarrollo de la educación virtual, tanto en la organización de la estructura general de la ruta como en sus características educativas.

\section{Fundamentos del diseño de la ruta de apropiación}

Los procesos de formación que utilizan las TIC como medio para movilizar la enseñanza-aprendizaje deben plantearse desde su conceptualización, diseño e implementación, bajo orientaciones pedagógicas y didácticas claras y flexibles que le permitan al estudiante acercarse al conocimiento de forma autónoma, en consonancia con el propósito de aprendizaje. En este sentido, el diseño de la ruta de apropiación de las TIC se sustenta en el marco teórico del TPACK (Mishra y Koehler, 2008), que hace referencia al conocimiento profesional del docente cuando establece relaciones entre el conocimiento tecnológico, pedagógico y disciplinar. Los estudios en esta perspectiva son variados (Cabero-Almenara, Marín-Díaz y Castaño-Garrido, 2015; Cejas-León, Navío-Gámez y Barroso-Osuna, 2016) y dieron sustento para que este proyecto de investigación en particular se centrara en los dominios derivados de las intersecciones que propone el modelo, como se muestra en la figura 1. 
Figura 1. Intersecciones de los dominios sobre los cuales se trabaja la ruta

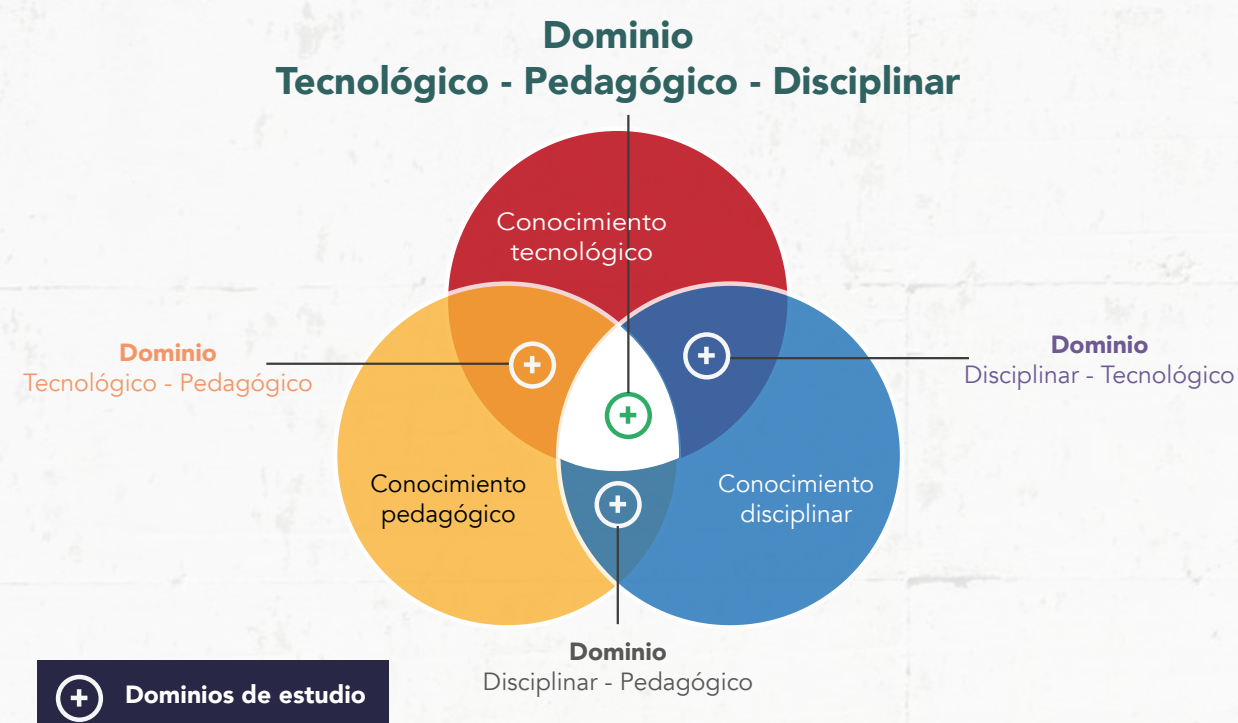

Fuente. Elaboración propia.

Desde esta perspectiva, las interacciones entre los diferentes conocimientos se definen de la siguiente manera:

- Disciplinar-pedagógico (DP): establece diálogos entre los conceptos propios de las áreas del conocimiento y sus posibilidades pedagógicas.

- Disciplinar-tecnológico (DT): se centra en conocer cómo utilizar la tecnología en el área disciplinar, independientemente de la enseñanza; es decir, en los campos laboral, investigativo y creativo.
- Pedagógico-tecnológico (PT): aborda conceptos generales sobre pedagogía, didáctica y su nexo con las TIC. Comprende todas aquellas actividades pedagógicas generales de cualquier área disciplinar que un profesor puede realizar utilizando tecnologías.

- Disciplinar-pedagógico-tecnológico (TPACK): en este convergen los conocimientos disciplinares, pedagógicos y tecnológicos, con el propósito de facilitar la creación de conocimiento de la disciplina. 
En este sentido, para definir los objetivos de estos dominios en la ruta, se revisaron diferentes documentos sobre formación en TIC para el desarrollo profesional docente, como lo son los de la Organización de las Naciones Unidas para la Educación, la Ciencia y la Cultura (Unesco), la Certificación Internacional de Competencias Digitales (ICDL) y del Ministerio de Educación Nacional (MEN). A partir de este análisis y clasificación, se definieron los objetivos y se revisó la manera como progresa cada uno según el nivel de complejidad, teniendo en cuenta la taxonomía de Bloom para la era digital (Churches, 2009).

A partir de los análisis, se establecieron tres niveles: iniciación, inmersión y profundización. En el nivel de iniciación, se hace énfasis en habilidades de conocimiento como recordar y comprender; en el de inmersión, en aplicar y analizar, y en el de profundización, en las habilidades de evaluar y crear. Así mismo, se clasificaron los objetivos por dominio disciplinar y por grado de progresión de estos en los tres niveles propuestos, teniendo en cuenta los saberes conceptuales, procedimentales y actitudinales que se deben fortalecer en los profesores universitarios para el uso transformador y alternativo de las TIC.

En sintonía con lo anterior, Gros (2011) plantea las generaciones por las cuales ha pasado la formación virtual, a saber, una primera centrada en materiales, una segunda centrada en gestores y plataformas virtuales, y una tercera basada en las actividades del estudiante. En el diseño de la ruta se opta por ésta última.
Retomando la definición de Cabero y Román (2006, p. 25) de las e-actividades como aquellas actividades que «son presentadas, realizadas o transferidas a través de la red», en esta ruta se propone centrar la formación en estas. De esta manera, las e-actividades se utilizarán para propiciar la participación activa del docente en la construcción de contenidos, a través de los diferentes recursos que ofrece la tecnología con actividades de encaje, rompecabezas, completar, arrastre para armar mapas conceptuales y otros organizadores gráficos y crucigramas, entre otros. Además, se propondrán e-actividades para el aprendizaje y la evaluación, utilizando estrategias tradicionales en ambientes virtuales, como bases de datos, wikis y foros, entre otros, además de actividades gamificadas.

Dichas e-actividades se propondrán tanto en la modalidad individual como en la colaborativa, por considerar que el aprendizaje, en el ámbito de la formación de profesores y de la docencia como profesión, exige el fortalecimiento de cada individuo, pero, a la vez, la necesidad de comprender que el conocimiento se construye en colectivos a través de redes disciplinares e interdisciplinares, que demandan en cada participante una actitud diferente frente al trabajo en equipo y la construcción con otros.

\section{Estructura general de la ruta de apropiación de TIC}

El entorno de aprendizaje de la ruta se diseñó bajo un enfoque metodológico disciplinar, es decir, se dio un lugar preponderante a los objetos de conocimiento de las 
diferentes áreas de saber, en tanto existen formas propias de representación, así como una pedagogía y didáctica específicas para cada una, y tecnologías propias en cada disciplina. Las áreas seleccionadas para el desarrollo de la ruta fueron salud, ingeniería y educación, al evidenciar la intención de participación de docentes de universidades en el ámbito regional, una vez enviada la invitación. Dichos saberes disciplinares se pondrán en diálogo con los saberes pedagógicos, ya que es necesario repensar los conocimientos teóricos y prácticos acerca de la pedagogía, puesto que estos no pueden dejarse al margen cuando se aborda un proceso de apropiación de las TIC en el contexto educativo. En este sentido, la propuesta de la ruta desde el diseño educativo privilegia el diálogo entre estos saberes.
Con base tanto en los hallazgos de la revisión de literatura —realizada en la fase inicial de la investigación-como en la experiencia del equipo interdisciplinar, se realizó un diseño global a partir de la estructura general que se deriva del modelo TPACK. Para responder a las tres áreas seleccionadas — salud, ingeniería y educación- se propusieron tres diplomados, que comparten algunos componentes y espacios comunes. Cada diplomado se propone con una duración de cien horas y una estructura de seis componentes que los participantes deben estudiar de manera gradual, tal como se ilustra en la figura 2, cada uno con una dedicación de estudio sugerida acorde con las habilidades y conocimientos que se espera propiciar.

Figura 2. Estructura del diplomado

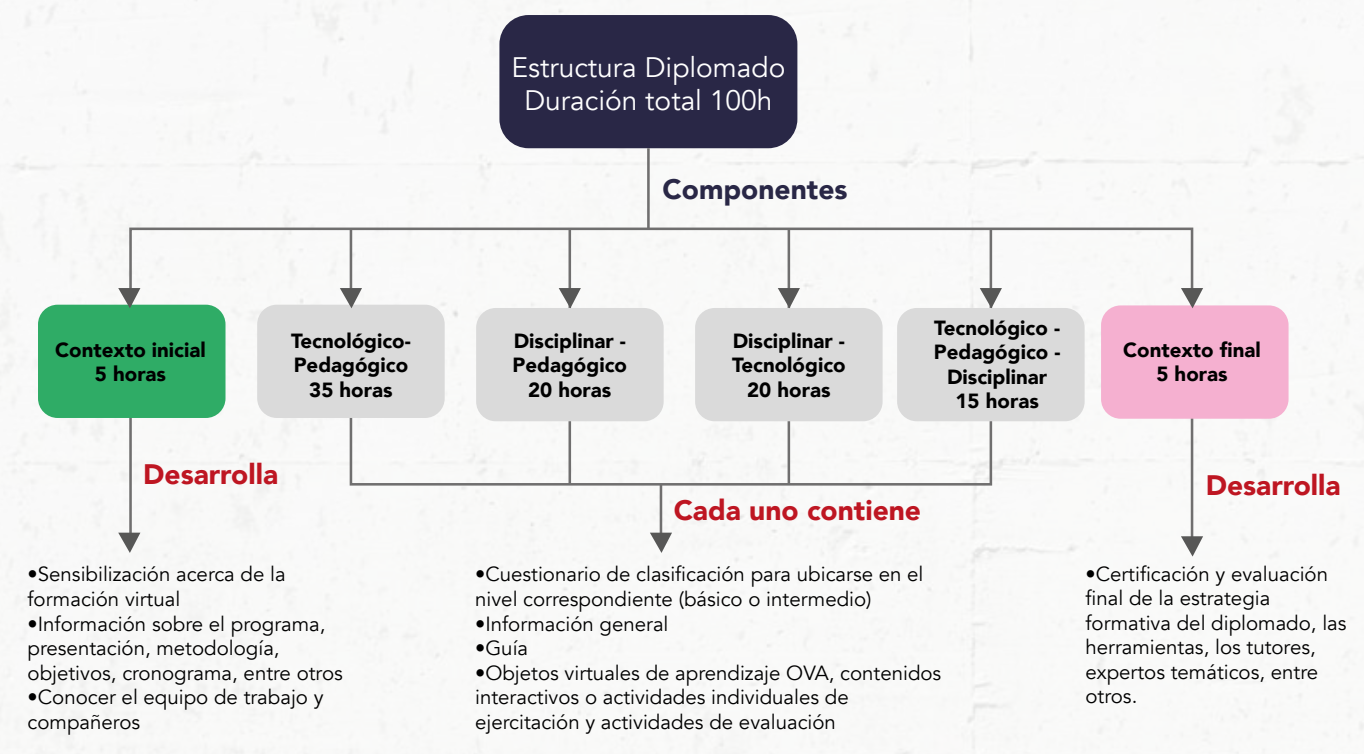

Fuente. Elaboración propia. 
Los componentes correspondientes a los dominios de conocimiento pedagógico-tecnológico, disciplinar-pedagógico, disciplinar-tecnológico y tecnológico-pedagógico-disciplinar contienen una información general - presentación, metodología del dominio de conocimiento, objetivo, cronograma y evaluación-. Además, contempla el desarrollo de los tres niveles, los cuales a su vez cuentan con presentación y objetivo, una guía general, recursos y e-actividades individuales en los niveles de iniciación e inmersión, y colaborativas, en el de profundización. A continuación, se ilustra de manera general cómo se estructura cada diplomado para las tres áreas disciplinares.

Respecto a las e-actividades propuestas para cada nivel, se determinó que pueden contener actividades individuales de ejercitación o evaluativas, y se intentó que en su mayoría fueran autogestionables, es decir, que el sistema les arroje el resultado, puesto que se busca que dicha ruta sea de uso libre y pueda desarrollarse sin la presencia de un docente. Para llegar a la actividad de trabajo colaborativo, correspondiente al nivel de profundización, los participantes deben realizar todas las actividades individuales de los niveles de iniciación e inmersión. Así mismo, para poder realizar el componente TPACK, es necesario que el participante apruebe los otros tres.

En términos generales, la duración de las e-actividades puede variar según si son de conceptualización, las cuales están organizadas en bloques de contenidos que no excedan 45 minutos, o de evaluación, que se estima en un tiempo promedio de una hora. Las de trabajo colaborativo tienen una duración máxima de 8 horas.

\section{Características educativas del diseño de la ruta}

Con base en el diseño general para la creación de los contenidos y las e-actividades, se trabajó inicialmente con un equipo de expertos y docentes universitarios con conocimiento sobre la didáctica de la enseñanza en las diferentes áreas disciplinares elegidas para la ruta. Su rol fundamental no era transmitir contenidos, sino proponer e-actividades para generar un entorno virtual de aprendizaje que facilitara la implicación y motivación de los participantes en la ruta, como:

- Fomentar un cambio en el saber, hacer o ser del docente participante.

- Privilegiar la actividad y participación del docente en la construcción de conceptos en función del aprendizaje activo, sobre la asimilación pasiva del conocimiento.

- Potenciar en el docente participante el aprendizaje individual y colaborativo.

- Aprovechar las potencialidades de las TIC para estimular la reflexión, autonomía, interactividad y motivación de los participantes.

Con base en las diversas características y condiciones definidas hasta el momento, dentro del diseño de la ruta se propone utilizar los recursos y realizar las actividades que se dispondrán, a través de Objetos Virtuales de Aprendizaje (OVA), así como recursos y actividades gamificadas, en 
tanto permiten articular de forma didáctica los propósitos educativos de la ruta. A continuación, se describen estos dos tipos de recursos.

\subsection{Objetos Virtuales de Aprendizaje}

Los OVA constituyen un conjunto de recursos digitales autocontenibles, granulares, interoperables, accesibles, durables en el tiempo y reutilizables, con un propósito educativo y constituidos por al menos tres componentes internos: contenidos, actividades de aprendizaje y elementos de contextualización (MEN, 2006). Algunos de los criterios pedagógicos y didácticos que se tuvieron en cuenta para utilizar los OVA en el desarrollo de la ruta de formación son:

- Propician la asimilación de conceptos, la generación de conocimiento y las estructuras de pensamiento.

- Promueven el constructivismo, manifestándose en el aprendizaje autónomo, el ejercicio de análisis de casos y el pensamiento crítico (Cabero et al., 2015, p. 6).

- Desarrollan habilidades de pensamiento crítico en la medida en que se relacionan los conocimientos previos con los nuevos, lo que da lugar al aprendizaje significativo.

- Posibilitan el aprendizaje individual y el colaborativo.

Dentro de los elementos del diseño instruccional que debe contener cada OVA para contextualizar a quien lo aborde, se proponen: componente disciplinar, nivel, objetivo, modalidad, descripción general, tiempo estimado, recursos disponibles, indicaciones específicas, referencias bibliográficas y elementos de ayuda para la autoevaluación -rúbrica, lista de chequeo-.

\subsection{Recursos y actividades gamificadas}

La integración de los elementos del juego a procesos existentes es lo que se ha denominado "gamificación» (Zichermann y Cunningham, 2011). En el diseño de la ruta está presente como una de las estrategias didácticas que permiten favorecer los procesos de formación a partir de la integración de estrategias lúdicas que fomentan la motivación y el aprendizaje, ya que la gamificación ayuda a generar escenarios atractivos para los estudiantes. Según Cook (2013), existen tres premisas principales que determinan la posibilidad de transformar un proceso, cualquiera que este sea, en un juego: que la actividad pueda ser aprendida, que las acciones del usuario puedan ser medidas y que las retroalimentaciones puedan ser entregadas de forma oportuna al usuario.

Los juegos que hacen parte de la ruta fueron desarrollados por equipo de investigación y son propuestos para el nivel de profundización de los diplomados en los componentes de Tecnologías en Educación, Tecnologías en Salud y Tecnología en Ingeniería. Estos son:

- Estrate-TIC: los jugadores deben resolver casos a partir del uso de las $\mathrm{TIC}$, utilizando un sistema de cartas. Cada participante tiene un rol en el equipo y es de vital importancia que trabajen en conjunto para encontrar la combinación de cartas adecuada para resolver el caso. 
- Smart City: usando tecnologías y políticas representadas en fichas, los participantes deben encontrar un equilibrio para mantener y generar nuevos recursos, $y$, de esta manera, construir una ciudad inteligente.

- Pandemia: simula un escenario de pandemia mundial, donde un grupo de expertos, que corresponde a un grupo de estudiantes, debe usar tecnologías y buscar las acciones adecuadas para combatir la pandemia y salvar a la humanidad.

A continuación, se mencionan otras características generales tenidas en cuenta en el diseño educativo de la ruta:

- Disponer una barra de progreso para que los participantes puedan consultar su avance general y en cada componente.

- Realizar encuentros sincrónicos para socializar los avances durante el desarrollo de la ruta.

- Desarrollar en el último nivel TPACK un trabajo colaborativo con los participantes de las diferentes áreas de conocimiento que propicie el trabajo interdisciplinar.

- Permitir de manera libre la conformación de los equipos en cada una de las actividades que lo requieran, propiciando la definición de roles para realizarla, lo cual exige que cada uno asuma un compromiso y reconozca la importancia de hacer su trabajo para contribuir a lograr los objetivos.
- Proponer la autoevaluación de la participación y desempeño en las diferentes actividades evaluativas.

\section{Conclusiones}

Realizar el diseño educativo de esta ruta de apropiación de TIC implica una reflexión a fondo sobre la relación entre lo disciplinar, lo educativo y lo tecnológico, lo cual lleva a preguntarse sobre cuáles son las transformaciones que acontecen en la educación cuando se utilizan las TIC. Esta ruta permite un acercamiento a las condiciones de nuestro contexto local que posibiliten una reflexión acerca de cómo y desde qué corriente pedagógica y didáctica se realizan las actuales prácticas educativas en las diferentes áreas disciplinares, y cómo la tecnología participa en este proceso. Todo esto sobre la base de que utilizar nuevas herramientas, sin remover las viejas estructuras, conlleva un uso limitado de las tecnologías (Garrido, 2005),

Si bien dentro del diseño de la ruta se pueden determinar sus características generales, solo en el desarrollo de los recursos y las actividades es posible poner en evidencia las demandas respecto a lo disciplinar, es decir, que las áreas de saber contienen en su interior diversidad de disciplinas con sus propias particularidades. En el área de la salud, por ejemplo, se encuentran disciplinas como Enfermería, Instrumentación Quirúrgica, Medicina, Odontología y Psicología, entre otras, que demandan estrategias, actividades y contenidos específicos. 
Estructurar los diplomados a partir de OVA ha permitido crear unidades de aprendizaje completas e independientes, lo cual hace posible secuenciar las acciones formativas hacia niveles más profundos de comprensión, reflexión y apropiación. Así mismo, el diseño de e-actividades ha significado un aporte para repensar la educación en ambientes virtuales de aprendizaje, pues estas permiten pasar de una acción expositiva hacia formas más dialógicas e interactivas.

\section{Referencias}

Cabero, J.; Román, P. (Coord.) (2006). E-actividades. Un referente básico para la formación en internet. Sevilla: Editorial MAD.

Cabero-Almenara, J.; Marín-Díaz, V.; Castaño-Garrido, C. (2015). Validación de la aplicación del modelo TPACK para la formación del profesorado en TIC. TIC Revista d'innovació educativa, (14), 13-22. DOI: https://doi.org/10.7203/ attic. 14.4001

Cejas-León, R.; Navío-Gámez, A.; Barroso-Osuna, J. M. (2016). Las competencias del profesorado universitario desde el modelo TPACK (conocimiento tecnológico y pedagógico del contenido). Píxel-Bit Revista de Medios y Educación, (49), 105-119. DOI: https://doi.org/10.12795/pixelbit.2016.i49.07

Churches, A. (2009). Taxonomía de Bloom para la era digital. Eduteka. Recuperado de https://bit.ly/2KjsrKf

Cook, W. (mayo de 2013). Five reasons you can't ignore gamification. Chief Learning Officer. Recuperado de https://bit.ly/3nuM2p5
Garrido, M. F. (2005). Formación basada en las Tecnologías de la Información y Comunicación: análisis didáctico del proceso de enseñanza-aprendizaje (Tesis doctoral). Universitat Rovira i Virgili, Tarragona, España. Recuperado de http://hdl.handle. net/10803/8909

Gros, B. (2011). Evolución y retos de la educación virtual. Construyendo el e-learning del siglo XXI. Barcelona: Editorial UOC.

Jiménez-Narváez, M.; Lopera-Pérez, M.; Arias, V.; Valderrama, A.; Ospina, D.; Bernal, D.; Segura, H. (2019). Formación docente universitaria en la modalidad de educación virtual. Ponencia presentada en el XXII Congreso linternacional Edutec. Edutec, Lima, Perú, 23-25 de octubre.

Koehler, M. J.; Mishra, P.; Cain, W. (2015). ¿Qué son los saberes tecnológicos y pedagógicos del contenido (TPACK)? Virtualidad, educación y ciencia, 6(10), 9-23.

Mishra, P.; Koehler, M. J. (2008). Introducing technological pedagogical content knowledge. Annual meeting of the American Educational Research Association. Nueva York, EE.UU., 24-28 de marzo.

Zichermann, G.; Cunningham, C. (2011). Gamification by design: Implementing game mechanics in web and mobile apps. Sebastopol, CA: O'Reilly Media. 